Kinetics of acid-induced degradation of tetra- and dihydrobiopterin in relation to their relevance as biomarkers of endothelial function

Mortensen, Alan; Lykkesfeldt, Jens

Published in:

Biomarkers

DOI:

$10.3109 / 1354750 X .2012 .730552$

Publication date:

2013

Document version

Early version, also known as pre-print

Citation for published version (APA):

Mortensen, A., \& Lykkesfeldt, J. (2013). Kinetics of acid-induced degradation of tetra- and dihydrobiopterin in relation to their relevance as biomarkers of endothelial function. Biomarkers, 18(1), 55-62.

https://doi.org/10.3109/1354750X.2012.730552 


\title{
Kinetics of acid-induced degradation of tetra- and dihydrobiopterin in relation to their relevance as biomarkers of endothelial function
}

\author{
Alan Mortensen and Jens Lykkesfeldt \\ Faculty of Health and Medical Sciences, Department of Veterinary Disease Biology, University of Copenhagen, \\ Copenhagen, Denmark \\ Abstract \\ The ratio of the nitric oxide synthase (NOS) cofactor tetrahydrobiopterin $\left(\mathrm{BH}_{4}\right)$ to its oxidized form dihydrobiopterin \\ $\left(\mathrm{BH}_{2}\right)$ has been suggested as an index of endothelial dysfunction. Consequently, much effort has been put into \\ preserving the in vivo equilibrium between these labile analytes. In the present study, we conducted a series of \\ stability experiments in aqueous solutions and blood to identify the most appropriate way of stabilizing $\mathrm{BH}_{4}$ and $\mathrm{BH}_{2}$. \\ Based on our results, we are able to recommend that blood samples are immediately stabilized with dithioerythriol \\ and protein precipitation conducted using trichloroacetic acid (TCA). \\ Keywords: Dihydrobiopterin, tetrahydrobiopterin, stability, endothelial dysfunction, biomarker, blood sampling
}

\section{Introduction}

Tetrahydrobiopterin $\left(\mathrm{BH}_{4}\right)$ is a cofactor of the aromatic amino acid hydroxylases, the nitric oxide synthases (NOS) and the alkylglycerol monooxygenase (Werner et al., 2011). $\mathrm{BH}_{4}$ is easily oxidized to 7,8-dihydrobiopterin $\left(\mathrm{BH}_{2}\right)$ which may be further oxidized to biopterin. $\mathrm{BH}_{4}$ is essential for the production of nitric oxide, an endothelial vasorelaxing factor, by NOS and may hence serve as a biomarker of endothelial dysfunction. While $\mathrm{BH}_{2}$ may bind to NOS just like $\mathrm{BH}_{4}$ (Vásquez-Vivar et al., 2002; Crabtree et al., 2008), it does not support the formation of nitric oxide (Presta et al., 1998) and instead superoxide is formed (Vásquez-Vivar et al., 2002). Studies have suggested that the ratio of $\mathrm{BH}_{4}$ to $\mathrm{BH}_{2}$ correlates better with endothelial function than the absolute concentration of $\mathrm{BH}_{4}$ (Noguchi et al., 2011; Crabtree et al., 2008; Takeda et al., 2009; Kar \& Kavdia, 2011). Consequently, appropriate stabilization and analysis of both $\mathrm{BH}_{4}$ and $\mathrm{BH}_{2}$ is a prerequisite.

$\mathrm{BH}_{4}$ and $\mathrm{BH}_{2}$ in plasma are determined either directly or indirectly by high-performance liquid chromatography
(HPLC) employing electrochemical (Powers et al., 1988), fluorescence (Fukushima \& Nixon, 1980; Mochizuki et al., 2005) or mass spectrometry detection (Feillet et al., 2008). Prior to analysis plasma proteins are removed; a step most often accomplished by acid precipitation. It has long been known that the auto-oxidation of $\mathrm{BH}_{4}$ proceeds more rapidly at higher $\mathrm{pH}$ (Blair \& Pearson, 1973; Pearson \& Blair, 1975; Pearson, 1974; Berka et al., 2004) and at $\mathrm{pH}<3$ the rate is markedly reduced (Lyudnikova et al., 2009). However, when it comes to $\mathrm{BH}_{2}$, its stability at the low $\mathrm{pH}$ used to precipitate proteins is less wellstudied. Some authors observed degradation of $\mathrm{BH}_{2}$ in acid (Katoh \& Akino, 1966; Schircks et al., 1978) and at neutral pH (Fukushima \& Nixon, 1979), whereas others have found a high stability of $\mathrm{BH}_{2}$ in aqueous solution at neutral and acidic pH (Dántola et al., 2008; Maharaj et al., 1990; Heales et al., 1988).

Importantly, however, the previous stability studies of $\mathrm{BH}_{4}$ and $\mathrm{BH}_{2}$ have been performed at micromolar or higher concentration whereas the physiologically relevant concentration range is nanomolar. Also, 
the studies have not been performed using the acids commonly used for protein precipitation.

Here, we present the results of a stability study of $\mathrm{BH}_{4}$ and $\mathrm{BH}_{2}$ at nanomolar concentration in the presence of three acids (meta-phosphoric acid (MPA), perchloric acid (PA), and trichloroacetic acid (TCA)) commonly used for plasma protein precipitation. We also report on the stability at neutral $\mathrm{pH}$ and examined whether antioxidants may affect the stability of $\mathrm{BH}_{4}$ and $\mathrm{BH}_{2}$ in aqueous solution. Finally, we have studied the stability of biopterins in blood under various clinically relevant conditions.

\section{Methods}

\section{Materials}

7,8-Dihydro-L-biopterin, 5,6,7,8-tetrahydro-L-biopterin dihydrochloride and L-biopterin were from Shircks Laboratories (Jena, Switzerland). Tris(2-carboxyethyl) phosphine hydrochloride (TCEP), disodium ethylenediaminetetraacetatedihydrate (EDTA), acetic acid, TCA, citric acid monohydrate, methanol, potassium iodide, hydrochloric acid, sodium hydroxide, potassium dihydrogen phosphate, dipotassium hydrogen phosphate and sodium dihydrogen phosphate monohydrate were purchased from VWR - Bie\&Berntsen A/S (Herlev, Denmark). Ammonium acetate, trisodium citrate dihydrate, 1,4-dithioerythritol (DTE), MPA, PA (20\%), iodine and disodium hydrogen phosphate dihydrate were obtained from Sigma-Aldrich (Brøndby, Denmark). All solutions were made in Milli-Q water.

\section{Analysis}

The HPLC system consisted of an Agilent (Hørsholm, Denmark) 1100 thermostatted autosampler, an Agilent 1200 binary pump, an Agilent 1200 fluorescence detector (excitation wavelength $275 \mathrm{~nm}$ and emission wavelength $442 \mathrm{~nm}$ ), and an Ultimate 3000 column compartment from Dionex (Hvidovre, Denmark). Upstream of the fluorescence detector was placed an ESA-conditioning cell (5021A) running at $400 \mathrm{mV}$ (controlled by a CouloChem II detector from ESA (Hvidovre, Denmark)) in order to oxidize $\mathrm{BH}_{2}$ to the more strongly fluorescent biopterin (only used when analyzing the stability of $\mathrm{BH}_{2}$ ).

For the analysis of $\mathrm{BH}_{4}$ and $\mathrm{BH}_{2}$ a Symmetry C8 column $(250 \times 4.6 \mathrm{~mm}, 5 \mu \mathrm{m})$ from Waters (Hedehusene, Denmark) was used. The samples were eluted with (1) $92 \%$ aqueous buffer containing $50 \mu \mathrm{M}$ EDTA and $50 \mathrm{mM}$ ammonium acetate-acetic acid buffer at pH 5.1 and (2) $8 \%$ methanol. The column was thermostatted at $30^{\circ} \mathrm{C}$ and the eluent flow was $1 \mathrm{ml} / \mathrm{min}$.

A Synergi Polar column $(150 \times 4.6 \mathrm{~mm}, 4 \mu \mathrm{m})$ and a guard column $(4 \times 3.0 \mathrm{~mm})$ from Phenomenex (Værløse, Denmark) were used for analysis of blood samples. The samples were eluted with (1) 95\% aqueous buffer containing $100 \mu \mathrm{M}$ EDTA and $50 \mathrm{mM}$ potassium phosphate buffer at $\mathrm{pH} 6.73$ and (2) $5 \%$ methanol for $4 \mathrm{~min}$ followed by a gradient to $90 \% \mathrm{~B}$ to clean the column. The column was thermostatted at $35^{\circ} \mathrm{C}$ and the eluent flow was $1 \mathrm{ml} / \mathrm{min}$.
Customarily, $350 \mathrm{~nm}$ is used for excitation in biopterin analysis. However, using an excitation wavelength of $275 \mathrm{~nm}$ increases the signal around 3 times, thereby increasing the limits of detection and quantitation. The drawback of using $275 \mathrm{~nm}$ is the possibility of exciting more compounds, thereby increasing the number of detected compounds and the possibility of overlapping peaks in the chromatogram. With our method the separation of peaks was good when exciting at $275 \mathrm{~nm}$ in blood samples and actually did not improve when exciting at $350 \mathrm{~nm}$. Therefore, $275 \mathrm{~nm}$ was used throughout.

\section{$\mathrm{BH}_{2}$}

A $20 \mu \mathrm{M}$ stock solution of $\mathrm{BH}_{2}$ in Milli-Q water was stored at $-80^{\circ} \mathrm{C}$ and used within 2 weeks. The stock solution was diluted to $20 \mathrm{nM}$ in $50 \mathrm{mM}$ phosphate buffer at $\mathrm{pH} 7.4$ (with or without DTE or TCEP) or $50 \mathrm{mM}$ citrate buffers at pH 3.0, 4.0, 5.0, and 6.0 (with or without DTE or TCEP). Because TCEP is an acid, the $\mathrm{pH}$ of the buffer solutions containing TCEP was adjusted with sodium hydroxide to give the same $\mathrm{pH}$ as the buffer without TCEP. Solutions containing $20 \mathrm{nM}$ of $\mathrm{BH}_{2}$ in 5\% MPA (with or without DTE or TCEP) or 5\% TCA (with or without DTE or TCEP) or 3\% PA (with or without DTE or TCEP), which are the commonly used concentrations of acid used for protein precipitation, were also analyzed.

Samples were left in the thermostatted autosampler at $25^{\circ} \mathrm{C}$ in plastic vials until subjected to analysis. A new vial was used for each injection of $10 \mu \mathrm{l}$.

$\mathrm{BH}_{4}$ A $200 \mu \mathrm{M}$ stock solution of $\mathrm{BH}_{4}$ in Milli-Q water containing either $6.5 \mathrm{mM}$ DTE or TCEP was stored at $-80^{\circ} \mathrm{C}$ and used within 2 weeks. The stock solution was diluted to $20 \mathrm{nM}$ in $50 \mathrm{mM}$ phosphate buffer at $\mathrm{pH} 7.4$ (containing either DTE or TCEP). Because TCEP is an acid, the $\mathrm{pH}$ of the buffer solution containing TCEP was adjusted with sodium hydroxide to give the same $\mathrm{pH}$ as the buffer without TCEP. Solutions containing $20 \mathrm{nM}^{\circ} \mathrm{BH}_{4}$ in $5 \%$ MPA (containing either DTE or TCEP) or 5\% TCA (containing either DTE or TCEP) or 3\% PA (containing either DTE or TCEP) were also analyzed. The samples were wrapped in tin foil and stored at $25^{\circ} \mathrm{C}$ in a thermostatted oven. Aliquots were drawn at intervals and analyzed according to the iodine oxidation method of Fukushima and Nixon (1980) employing the same eluent and column as for the $\mathrm{BH}_{2}$ analysis.

\section{Blood samples}

Blood was drawn from a male volunteer in $4 \mathrm{ml}$ K3E BD Vacutainer tubes to which were added $100 \mu \mathrm{l} 4 \%$ DTE yielding a final concentration of DTE of around $0.1 \%$. Blood was divided in three aliquots. One aliquot was left in a thermostatic oven at $25^{\circ} \mathrm{C}$ in the vacutainer. A second aliquot was centrifuged, the plasma separated and placed in the oven. A third aliquot was centrifuged and the plasma divided into three. The three fractions were added TCA, MPA, or PA: to four volumes of plasma was added one volume of $1 \mathrm{M}$ TCA, $25 \%$ MPA or $15 \%$ PA. The 
samples were spun and the supernatant stored in the oven together with the other samples.

Aliquots were taken regularly and analyzed by the method of Fukushima and Nixon (1980). Blood was centrifuged, plasma separated and proteins precipitated with TCA, MPA, or PA before oxidation with iodine. Plasma was added TCA, MPA, or PA.

\section{Results}

$\mathrm{BH}_{2}$ Regardless of $\mathrm{pH}$, the major detected degradation product of $\mathrm{BH}_{2}$ was biopterin. Mass balance analysis showed that biopterin could not be the only compound formed from degradation of $\mathrm{BH}_{2}$ (see below), however, biopterin was the only detectable degradation product of significance. Figure $1 \mathrm{~A}$ shows the chromatogram of $\mathrm{BH}_{2}$ in phosphate buffer after $108 \mathrm{~h}$. The peak at $5 \mathrm{~min}$ is $\mathrm{BH}_{2}$ whereas the peak at $5.36 \mathrm{~min}$ is biopterin. A number of very small peaks could also be discerned. It was not the purpose of this study to precisely elucidate the reaction mechanisms and determine all degradation products.

Degradation of $\mathrm{BH}_{2}$ followed first-order kinetics, i.e. exponential loss of $\mathrm{BH}_{2}$ in all solutions examined. The first-order rate constants are presented in Table 1. These rate constants are in reality not true first-order rate constants, as they depend on $\mathrm{pH}$ and may also depend on oxygen concentration (see Discussion section). However, the concentration of hydrogen ions can be considered to be constant over time in the solutions, either because the solutions are buffered or because of a large surplus of hydrogen ions compared to $\mathrm{BH}_{2}$ in the acidic solutions.
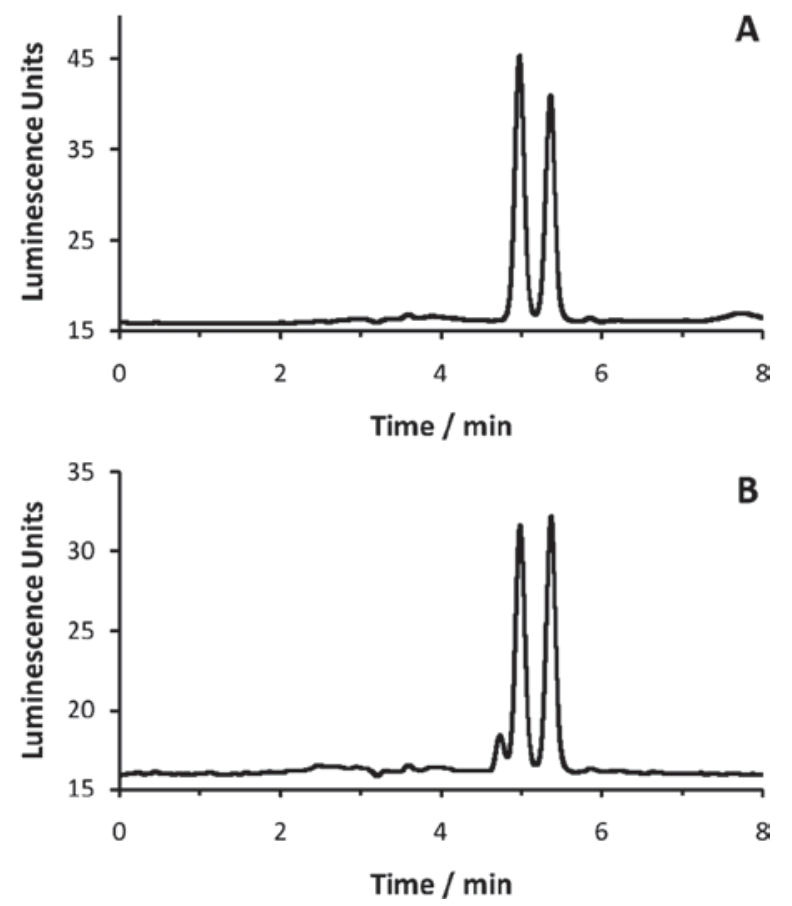

Figure 1. Chromatograms of dihydrobiopterin $\left(\mathrm{BH}_{2}\right)$ in phosphate buffer ( $\mathrm{pH}$ 7.4) (A) without and with (B) dithioerythritol (DTE) after $108 \mathrm{~h}$.
Likewise, the concentration of oxygen is much higher than the concentration of $\mathrm{BH}_{2}$ and can be considered to remain constant throughout the course of reaction as well.

$\mathrm{BH}_{2}$ is rather stable in solution with half-lives of $>2.5 \mathrm{~h}$ regardless of $\mathrm{pH}$. However, $\mathrm{pH}$ is not the only determinant of $\mathrm{BH}_{2}$ stability. Somewhat surprisingly, the rate of degradation of $\mathrm{BH}_{2}$ is lower in PA than in TCA and MPA, despite the lower $\mathrm{pH}$ and despite the fact that $\mathrm{PA}$, besides being a strong acid, is also an oxidizing acid that potentially could oxidize $\mathrm{BH}_{2}$. The rate of degradation is also higher in MPA than in TCA despite the higher $\mathrm{pH}$ in the former. Comparing the phosphate buffer at $\mathrm{pH} 7.4$ and the citrate buffer at $\mathrm{pH} 6.0$, the higher $\mathrm{pH}$ of the phosphate buffer leads to an increase in reaction rate rather than a decrease.

From the rate constants in Table 1, it is clear that the rate of degradation of $\mathrm{BH}_{2}$ is not proportional to the hydrogen ion concentration, but the (apparent) firstorder rate constant shows a weaker dependence on acidity. Looking at the citrate buffers alone, the first-order rate constants could be shown to perfectly follow a secondorder polynomial expression in the $\mathrm{pH}$ range 3-6:

$$
\begin{gathered}
k=0.01 \times(\mathrm{pH})^{2}-0.12 \times \mathrm{pH}+0.38\left(\mathrm{BH}_{2} \text { alone }\right) \\
k=0.0055 \times(\mathrm{pH})^{2}-0.076 \times \mathrm{pH}+0.26\left(\mathrm{BH}_{2} \text { with TCEP }\right) \\
k=0.0059 \times(\mathrm{pH})^{2}-0.086 \times \mathrm{pH}+0.31\left(\mathrm{BH}_{2} \text { with DTE }\right)
\end{gathered}
$$

These mathematical relationships indicate that the mechanism of degradation is not merely a matter of protonation of $\mathrm{BH}_{2}$, followed by oxidation of the protonated $\mathrm{BH}_{2}$, the $\mathrm{pK}_{\mathrm{a}}$ of which has been determined to be 2.56 (Maharaj et al., 1990).

Since the major pathway of degradation of $\mathrm{BH}_{2}$ in neutral and acidic solution is oxidation, it is relevant to examine if antioxidants could stabilize $\mathrm{BH}_{2}$. DTE and the closely related dithiothreitol (DTT) are often used to stabilize $\mathrm{BH}_{4}$ in plasma before analysis. A concentration of $0.1 \%$, corresponding to $6.5 \mathrm{mM}$, is commonly employed at which concentration DTT has been found to be able to stabilize $\mathrm{BH}_{4}$ in plasma for a few hours (Fekkes \& Voskuilen-Kooijman, 2007). TCEP is an antioxidant used primarily for reducing disulfide bonds. However, it has been succesfully used for stabilizing ascorbic acid in solution (Lykkesfeldt, 2000) and we therefore decided to test its suitability with respect to biopterins. The same concentration, i.e. $6.5 \mathrm{mM}$, of TCEP was used throughout.

Neither of the two antioxidants was very effective in protecting $\mathrm{BH}_{2}$ from oxidation (Table 1). In solutions of the three acids, both antioxidants conferred the same level of modest protection to $\mathrm{BH}_{2}$. As far as DTE goes, this is somewhat surprising, as the antioxidative capability is considered to be lost at low $\mathrm{pH}$. In citrate and phosphate buffers, TCEP does provide some protection whereas DTE does not. In fact, DTE seems to have a pro-oxidant effect at 
Table 1. First-order rate constants $\left(\mathrm{h}^{-1}\right)$ for degradation of $\mathrm{BH}_{2}$.

\begin{tabular}{llccc}
\hline Acid/buffer & $\mathrm{pH}$ & $\mathrm{BH}_{2}$ & $\mathrm{BH}_{2}+\mathrm{TCEP}$ & $\mathrm{BH}_{2}+\mathrm{DTE}$ \\
\hline PA & 0.75 & $0.0943 \pm 0.0022$ & $0.0786 \pm 0.0011$ & $0.0828 \pm 0.0013$ \\
TCA & 0.89 & $0.2112 \pm 0.0038$ & $0.1475 \pm 0.0013$ & $0.1464 \pm 0.0011$ \\
MPA & 1.51 & $0.2701 \pm 0.0058$ & $0.2434 \pm 0.0023$ & $0.2441 \pm 0.0017$ \\
Citrate & 3.0 & $0.1050 \pm 0.0034$ & $0.0814 \pm 0.0004$ & $0.1037 \pm 0.0007$ \\
Citrate & 4.0 & $0.0496 \pm 0.0011$ & $0.0454 \pm 0.0005$ & $0.0587 \pm 0.0007$ \\
Citrate & 5.0 & $0.0216 \pm 0.0009$ & $0.0170 \pm 0.0003$ & $0.0273 \pm 0.0004$ \\
Citrate & 6.0 & $0.0061 \pm 0.0005$ & $0.0028 \pm 0.0003$ & $0.0060 \pm 0.0003$ \\
Phosphate & 7.4 & $0.0087 \pm 0.0008$ & $0.0050 \pm 0.0003$ & $0.0112 \pm 0.0003$ \\
\hline
\end{tabular}

$\mathrm{BH}_{2}$, dihydrobiopterin; DTE, 1,4-dithioerythritol; TCEP, tris(2-carboxyethyl)phosphine hydrochloride.

$\mathrm{pH} 4,5$, and 7.4 (Table 1). Besides affecting the rate of degradation of $\mathrm{BH}_{2}$, DTE also affected the reaction pathway. In Figure 1B it can be seen that DTE caused formation of a compound eluting at $4.73 \mathrm{~min}$. This compound was observed in citrate and phosphate buffers but not in the more acidic solutions. It was virtually absent in solutions of $\mathrm{BH}_{2}$ without antioxidants and in solutions with TCEP. The nature of this compound remains to be established. At $\mathrm{pH} 7.4$ after $108 \mathrm{~h}$ of autoxidation, $\mathrm{BH}_{2}$ and biopterin accounted for $100 \%$ of the added $\mathrm{BH}_{2}$. With TCEP, $\mathrm{BH}_{2}$, and biopterin also accounted for the majority of species present (around 94\%) after 108h. However, in the presence of DTE at $\mathrm{pH} 7.4$, the concentration of $\mathrm{BH}_{2}$ and biopterin only amounted to $30 \%$ each, that is, $40 \%$ of the initial amount of $\mathrm{BH}_{2}$ had been converted to compound(s) other than biopterin, one of them the species at $4.73 \mathrm{~min}$. In general, regardless of $\mathrm{pH}$ more $\mathrm{BH}_{2}$ could be recovered as either $\mathrm{BH}_{2}$ or biopterin at the end of the reaction if no antioxidant was used, whereas DTE gave a low recovery of $\mathrm{BH}_{2}$ as either $\mathrm{BH}_{2}$ or biopterin; TCEP was in between (Table 2). Also, with the citrate buffers a lower amount of $\mathrm{BH}_{2}$ was recovered as either $\mathrm{BH}_{2}$ or biopterin at low $\mathrm{pH}$ compared to high. However, phosphate buffer provided a lower recovery of $\mathrm{BH}_{2}$ as either $\mathrm{BH}_{2}$ or biopterin than did citrate buffer at $\mathrm{pH} 6$ once again demonstrating that $\mathrm{pH}$ is not the only factor influencing conversion of $\mathrm{BH}_{2}$. A particularly low recovery was observed with MPA (43\% with antioxidant and $54 \%$ without). Besides the small peak at 4.73 min observed at high $\mathrm{pH}$ with DTE (Figure 1), no other peaks (except for very small ones) than $\mathrm{BH}_{2}$ and biopterin were detected meaning that $\mathrm{BH}_{2}$ was converted into nonfluorescent compounds.

\section{$\mathrm{BH}_{4}$}

The stability of $\mathrm{BH}_{4}$ in the three acids was also studied. Because of its instability, $\mathrm{BH}_{4}$ stability was only examined in the presence of antioxidant-in clinical settings, DTE or DTT is routinely added to the sample to avoid degradation.

$\mathrm{BH}_{4}$ was remarkably stable in all three acids with DTE or TCEP. Over a period of $48 \mathrm{~h}$ no statistically significant degradation took place with one exception: $\mathrm{BH}_{4}$ in TCA with DTE ( $p<0.001$, linear regression) (Figure 2$)$. From the data, it was not evident whether the degradation was zero- or first-order: zero-order rate constant
Table 2. Percent recovery of $\mathrm{BH}_{2}$ as either $\mathrm{BH}_{2}$ or biopterin at the end of the degradation.

\begin{tabular}{lccc}
\hline & Without antioxidant & TCEP & DTE \\
\hline PA & 71 & 67 & 71 \\
TCA & 81 & 66 & 66 \\
MPA & 54 & 43 & 43 \\
Citrate pH 3 & 69 & 61 & 53 \\
Citrate pH 4 & 79 & 70 & 54 \\
Citrate pH 5 & 86 & 80 & 57 \\
Citrate pH 6 & 100 & 99 & 83 \\
Phosphate pH 7.4 & 100 & 94 & 61 \\
\hline
\end{tabular}

$\mathrm{BH}_{2}$, dihydrobiopterin; DTE, 1,4-dithioerythritol; MPA, metaphosphoric acid; PA, perchloric acid; TCA, trichloroacetic acid; TCEP, tris(2-carboxyethyl)phosphine hydrochloride.

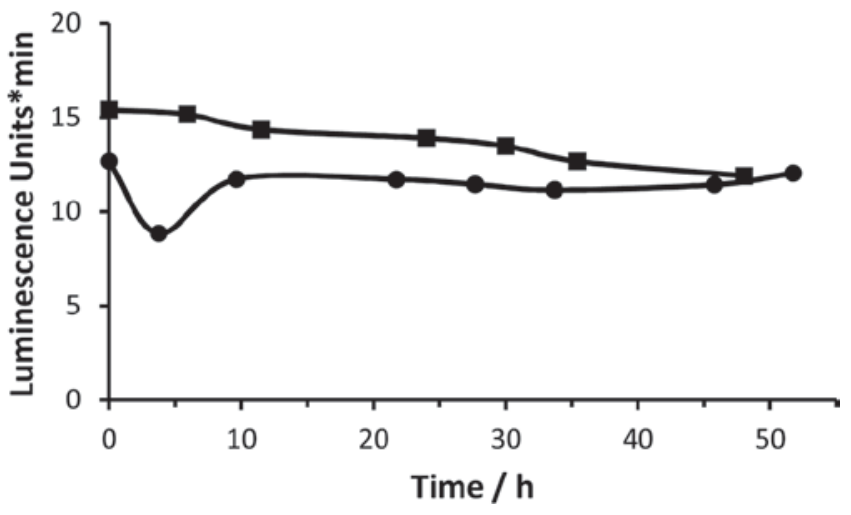

Figure 2. Degradation of tetrahydrobiopterin $\left(\mathrm{BH}_{4}\right)$ in trichloroacetic acid (TCA) with dithioerythritol (DTE) (closed square) and in meta-phosphoric acid (MPA) with DTE (closed circle).

$0.094 \pm 0.006 \mathrm{nM} / \mathrm{h}\left(R^{2}=0.977\right)$ or first-order rate constant $0.0053 \pm 0.0004 \mathrm{~h}^{-1}\left(R^{2}=0.973\right)$. Concomitantly, the level of oxidized biopterins $\left(\mathrm{BH}_{2}\right.$ and biopterin) rose. Another interesting finding was that the response of $\mathrm{BH}_{4}$ was around 20\% lower in MPA than in TCA and PA, regardless of whether DTE or TCEP was used. This lower response did not seem to be due to degradation as the concentration of $\mathrm{BH}_{4}$ in MPA did not change with time (Figure 2).

The stability of $\mathrm{BH}_{4}$ in phosphate buffer at $\mathrm{pH} 7.4$ was also studied. In Figure 3 is shown the degradation of $\mathrm{BH}_{4}$ as a function of time. In contrast to the situation at low $\mathrm{pH}$, $\mathrm{BH}_{4}$ stability at $\mathrm{pH} 7.4$ was markedly reduced. Apparently, the degradation of $\mathrm{BH}_{4}$ with DTE followed zero-order kinetics with a rate constant of $0.254 \pm 0.013 \mathrm{nM} / \mathrm{h}$, while 
the degradation in the presence of TCEP was first-order with a rate-constant of $0.145 \pm 0.025 \mathrm{~h}^{-1}$.

\section{Blood}

Stability of blood was only studied with DTE as the prior experiments with $\mathrm{BH}_{4}$ had indicated that DTE was a better antioxidant than TCEP at $\mathrm{pH}$ 7.4. Regardless in which state blood samples were left at $25^{\circ} \mathrm{C}$ (whole blood, plasma or acidified plasma) and which acid was used for precipitation, no significant changes in $\mathrm{BH}_{4}$ or $\mathrm{BH}_{2}$ levels or the $\mathrm{BH}_{2}$-to- $\mathrm{BH}_{4}$ ratio over time were found. However, there were considerable differences in analytical response or recovery. The data were analyzed by two-way ANOVA using the acid and pretreatment of the blood sample as factors followed by Tukey's post hoc test of planned comparisons. Table 3 shows the results with respect to acid used for precipitation. $\mathrm{BH}_{4}$ levels were 30-35\% lower when precipitating proteins with MPA compared to the two other acids. TCA gave a slightly higher $\mathrm{BH}_{4}$ level than did PA. The situation was reversed when it came to the oxidized biopterins $\mathrm{BH}_{2}$, and biopterin: here MPA gave the highest level and TCA the lowest level. Despite the higher level of $\mathrm{BH}_{2}+$ biopterin with MPA, it was not enough to offset the lower level of $\mathrm{BH}_{4}$ and the total level of biopterins was significantly reduced

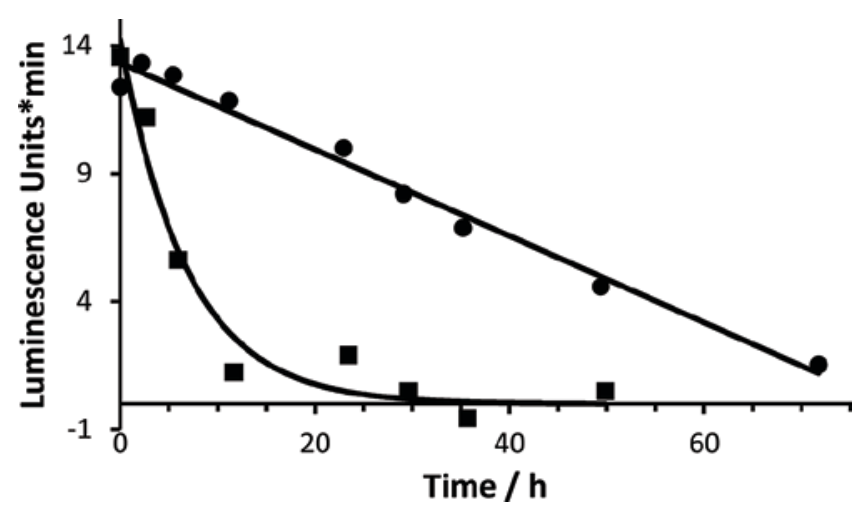

Figure 3. Degradation of tetrahydrobiopterin $\left(\mathrm{BH}_{4}\right)$ in phosphate buffer ( $\mathrm{pH}$ 7.4) with dithioerythritol (DTE) (closed circle) and tris(2-carboxyethyl)phosphine hydrochloride (TCEP) (closed square). compared to the two other acids; there was no difference between TCA and PA (Table 3).

When it comes to pretreatment of the samples, $\mathrm{BH}_{4}$ levels were unaffected by how the blood samples were stored (Table 4). $\mathrm{BH}_{2}+$ biopterin levels, however, were slightly lower in the acid-precipitated samples than in whole blood and plasma samples. This affected the $\mathrm{BH}_{2}-$ to- $\mathrm{BH}_{4}$ ratio but not the level of total biopterins.

\section{Discussion}

In the present study, we wanted to investigate the stability of $\mathrm{BH}_{4}$ and $\mathrm{BH}_{2}$ both under controlled aqueous conditions and more clinically relevant conditions in order to provide a rational basis for recommending a standard stabilization procedure which adequately preserves the in vivo equilibrium of the analytes.

With regard to $\mathrm{BH}_{2}$, the present study demonstrates-in line with previous findings - that $\mathrm{BH}_{2}$ is degraded in aqueous solution at neutral and acidic $\mathrm{pH}$, the major degradation product being biopterin. Katoh and Akino observed that $\mathrm{BH}_{2}$ in $0.5 \mathrm{~N}$ sulfuric acid (stored overnight at $-20^{\circ} \mathrm{C}$ ) was oxidized to biopterin and to a lesser extent dehydroxylated to form deoxysepiapterin (Katoh \& Akino, 1966). Likewise, $\mathrm{BH}_{2}$ in $25 \%$ acetic acid was found to be degraded to a number of compounds, the major compound being biopterin even when the reaction was carried out under nitrogen for $20 \mathrm{~min}$ at $60^{\circ} \mathrm{C}$ (Schircks et al., 1978). Formation of biopterin was believed to be due to intermolecular redox reactions. Fukushima and Nixon (1979) studied the autoxidation of $\mathrm{BH}_{2}$ at $\mathrm{pH} 4,6$, and 7.5. After 2-3 days of autoxidation at room temperature they found that biopterin was the major product with minor amounts of sepiapterin, deoxysepiapterin, and xanthopterin. None of these studies looked at the kinetics of degradation of $\mathrm{BH}_{2}$.

However, other studies failed to observe any degradation. Thus, Maharaj et al. found that the absorption spectrum of $\mathrm{BH}_{2}$ did not change with time (time span not indicated) at low $\mathrm{pH}(0.15 \mathrm{~N}$ and $5 \mathrm{~N} \mathrm{HCl})$ indicating that $\mathrm{BH}_{2}$ was stable under these conditions (Maharaj et al., 1990). The lack of any observable changes may have been due to a too short period of observation. Likewise,

Table 3. Plasma levels of biopterins with respect to acidification strategy.

\begin{tabular}{|c|c|c|c|c|}
\hline & $\mathrm{BH}_{4} / \mathrm{nM}$ & $\mathrm{BH}_{2}+$ Biopterin $/ \mathrm{nM}$ & Total/nM & $\mathrm{BH}_{2}-\mathrm{BH}_{4}$ ratio \\
\hline TCA & $24.9 \pm 1.0^{\mathrm{a}}$ & $9.2 \pm 1.3^{\mathrm{a}}$ & $34.1 \pm 1.0^{\mathrm{a}}$ & $0.37 \pm 0.06^{\mathrm{a}}$ \\
\hline MPA & $16.3 \pm 1.3^{\mathrm{b}, * * *}$ & $12.6 \pm 1.3^{\mathrm{b}, * * *}$ & $28.9 \pm 1.5^{\mathrm{b}, * * *}$ & $0.78 \pm 0.11^{\mathrm{b}, * * *}$ \\
\hline $\mathrm{PA}$ & $23.1 \pm 1.1^{\mathrm{c}, * * *}$ & $10.2 \pm 0.9^{\mathrm{c}, *}$ & $33.3 \pm 0.9^{\mathrm{a}}$ & $0.44 \pm 0.05^{\mathrm{c}, *}$ \\
\hline
\end{tabular}

Numbers bearing different superscript lettering are significantly different.

$\mathrm{BH}_{2}$, dihydrobiopterin; $\mathrm{BH}_{4}$, tetrahydrobiopterin; MPA, meta-phosphoric acid; $\mathrm{PA}$, perchloric acid; TCA, trichloroacetic acid. ${ }^{*} p<0.05$, ${ }^{* * *} p<$ 0.001 compared to TCA.

Table 4. Bench stability $\left(25^{\circ} \mathrm{C}\right)$ of biopterins under various conditions.

\begin{tabular}{|c|c|c|c|c|}
\hline & $\mathrm{BH}_{4} / \mathrm{nM}$ & $\mathrm{BH}_{2}+$ Biopterin/nM & Total/nM & $\mathrm{BH}_{2}-\mathrm{BH}_{4}$ ratio \\
\hline Blood & $21.0 \pm 4.0$ & $11.0 \pm 2.1^{\mathrm{a}}$ & $32.1 \pm 2.3$ & $0.57 \pm 0.23^{\mathrm{a}}$ \\
\hline Plasma & $21.5 \pm 3.9$ & $11.0 \pm 1.6^{\mathrm{a}}$ & $32.4 \pm 2.6$ & $0.54 \pm 0.19^{\mathrm{a}, \mathrm{b}}$ \\
\hline Acid-precipitated & $21.7 \pm 4.0$ & $10.0 \pm 1.7^{\mathrm{b} *}$ & $31.8 \pm 3.0$ & $0.49 \pm 0.17^{\mathrm{b} *}$ \\
\hline
\end{tabular}

Numbers within a column bearing different superscript lettering are significantly different.

$\mathrm{BH}_{2}$, dihydrobiopterin; $\mathrm{BH}_{4}$, tetrahydrobiopterin. ${ }^{*} p<0.05$. 
Heales et al. did not observe any degradation of $\mathrm{BH}_{2}$ at $\mathrm{pH}$ 1.6, 5.3, and 7.6 when autoxidized for $30 \mathrm{~min}$ (Heales et al., 1988). As shown here, $\mathrm{BH}_{2}$ is only degraded to a small extent in $30 \mathrm{~min}$. Davis et al. observed no degradation of $\mathrm{BH}_{2}$ for up to $150 \mathrm{~min}$ at $\mathrm{pH}$ 6.8-8.2 (Davis et al., 1988), which is in line with our results showing little degradation during this short-time span. On the other hand, Dántola et al. followed the degradation of $\mathrm{BH}_{2}$ at neutral $\mathrm{pH}$ at $25^{\circ} \mathrm{C}$ for 2 days and observed very little degradation (Dántola et al., 2008). They determined a first-order rate constant of degradation of $\mathrm{BH}_{2}$ of $0.0004 \mathrm{~h}^{-1}$ (this rate constant should be regarded with some caution as the reaction was only followed for $48 \mathrm{~h}$, during which very little reaction would have taken place), which is at least an order of magnitude lower than our results at $\mathrm{pH} 6.0$ and 7.4. They did not use buffers but adjusted $\mathrm{pH}$ to $7.0 \mathrm{using}$ hydrochloric acid or aqueous sodium hydroxide. In combination with our results, this could indicate that buffers actually enhance the degradation of $\mathrm{BH}_{2}$, in line with our observations that the rate of degradation depends on the nature of the buffer/acid and not only on $\mathrm{pH}$. That the nature of the buffer also plays a role in the degradation of $\mathrm{BH}_{2}$ (and $\mathrm{BH}_{4}$ ), has been demonstrated previously where it was found, that the degradation product profile was different in succinate, phosphate and Tris buffer at the same pH (Fukushima \& Nixon, 1979).

With regard to $\mathrm{BH}_{4}$, our results showed an increased stability of $\mathrm{BH}_{4}$ at low $\mathrm{pH}$ compared to neutral $\mathrm{pH}$, which is in line with previous findings. Autoxidation of $\mathrm{BH}_{4}$ proceeded more rapidly as $\mathrm{pH}$ was increased (Blair \& Pearson, 1973; Pearson \& Blair, 1975; Pearson, 1974). At high $\mathrm{pH}$, this is due to deprotonation of the uncharged $\mathrm{BH}_{4}\left(\mathrm{p} K_{\mathrm{a}} 10.5\right.$ (Blair \& Pearson, 1973)) leading to a negatively charged species that is easily oxidized. On the other hand, at lower $\mathrm{pH}$ singly ( $\mathrm{p} K_{\mathrm{a}} 5.6$ (Pfleiderer, 1982)) or even doubly charged species ( $\mathrm{p} K_{\mathrm{a}} 1.3$ (Pfleiderer, 1982)) are formed which are less susceptible toward oxidation. Thus, at $\mathrm{pH}<3$ the rate was markedly reduced and at $\mathrm{pH}$ 1-2 no degradation was observed during 20-min incubation (Lyudnikova et al., 2009). On the other hand, Howells and Hyland observed that $41 \mathrm{nM} \mathrm{BH}_{4}$ in $0.1 \mathrm{M} \mathrm{HCl}(\mathrm{pH}$ around 1) was completely oxidized to $\mathrm{BH}_{2}$ and biopterin in 15 min (Howells \& Hyland, 1987). Increasing the concentration to $415 \mathrm{nM}$ meant that only $1.3 \%$ was oxidized in $15 \mathrm{~min}$, and increasing the concentration even further led to an even lower percentage being oxidized (Howells \& Hyland, 1987), showing that results at micromolar (in the study by Lyudnikova et al. a concentration of around $0.1 \mathrm{mM}$ was employed) and millimolar concentrations cannot be used to predict the behavior at the physiologically relevant nanomolar range.

At higher $\mathrm{pH}$, the oxidation of $\mathrm{BH}_{4}$ is faster. The half-life of $\mathrm{BH}_{4}$ can be estimated to be around half an hour at $\mathrm{pH} 3$ and at $\mathrm{pH} 4$ and above almost complete oxidation takes place in 20 min (Lyudnikova et al., 2009). At pH 5.3-7.6 the half-life was estimated to around $9 \mathrm{~min}$ (Heales et al., 1988), and another study found half-lives of 6.5-60 $\mathrm{min}$ at pH 6.8-8.2 depending on the buffer used (Davis et al.,
1988). Similarly, a half-life of $15-20$ min was observed at pH 7.4 (Valent \& Tóth, 2006). Somewhat greater stability was found by Berka et al. (2004) who observed half-lives of 40 and $120 \mathrm{~min}$ at $\mathrm{pH} 7$ and 3, respectively.

$\mathrm{BH}_{4}$, being more reduced than $\mathrm{BH}_{2}$, is more prone to oxidation than $\mathrm{BH}_{2}$, and an antioxidant is almost always used when handling samples containing $\mathrm{BH}_{4}$. Co-administration of antioxidants greatly enhances the stability of $\mathrm{BH}_{4}$. Our studies showed no significant degradation of $\mathrm{BH}_{4}$ in acid with either DTE or TCEP for $48 \mathrm{~h}$ except for the combination TCA and DTE. Why $\mathrm{BH}_{4}$ showed less stability in TCA with DTE than in the two other acids is not known. A previous study has shown that acid alone was not enough to stabilize $\mathrm{BH}_{4}$ at nanomolar concentration (Howells \& Hyland, 1987), whereas at micromolar concentration acid without antioxidant was sufficient to stabilize $\mathrm{BH}_{4}$, at least for 20 min (Howells \& Hyland, 1987; Lyudnikova et al., 2009). Ascorbic acid $(5.7 \mathrm{mM})$ prevented oxidation of $31 \mu \mathrm{M} \mathrm{BH}_{4}$ for $20 \mathrm{~h}$ and $41.5 \mathrm{nM} \mathrm{BH}_{4}$ (both containing $0.1 \mathrm{M} \mathrm{HCl}$ ) for $4 \mathrm{~h}$ when the solutions were gassed with argon (Howells \& Hyland, 1987). In another study, it was found that $100 \mathrm{nM} \mathrm{BH}_{4}$ at pH 7.4 was completely oxidized within $20 \mathrm{~min}$ and that $100 \mu \mathrm{M}$ ascorbic acid increased the half-life to around 70 min (Heller et al., 2001). Berka et al. found that DTT was able to lower the decay rate fourfold at $\mathrm{pH} 7$, and that DTE together with DETAPAC was able to increase the half-life by a factor of 6 at pH 3 (Berka et al., 2004). Another study found that $0.5 \mathrm{mM}$ DTT inhibited the oxidation of $\mathrm{BH}_{4}$ at $\mathrm{pH} 7.4$ by $85-90 \%$ (Valent \& Tóth, 2006). Other studies have found that $0.1 \%$ DTE or $0.1 \%$ DTT is able to stabilize $\mathrm{BH}_{4}$ for $2.5-5 \mathrm{~h}$ when a chelating agent such as EDTA is also used (Cañada-Cañada et al., 2009; Espinosa-Mansilla et al., 2008; Powers et al., 1988; Howells et al., 1986). Our studies showed that degradation of $\mathrm{BH}_{4}$ at $\mathrm{pH} 7.4$ begins immediately and that there is not a lag phase during which no degradation takes place (Figure 3), as other studies might indicate. However, with a rate constant of $0.25 \mathrm{nM} \mathrm{h}^{-1}$ in DTE only $1 \%$ of $\mathrm{BH}_{4}$ would have degraded during the first hour.

The lower $\mathrm{BH}_{4}$ response with MPA remains unclear. It does not seem to be degradation as there is no change for $50 \mathrm{~h}$ (Figure 2), but interference with the assay, i.e. oxidation by iodine, cannot be ruled out.

Only few publications have reported on the stability of biopterins in blood samples. Fiege et al. studied the stability of $\mathrm{BH}_{4}$ in blood samples stored at $-80^{\circ} \mathrm{C}$ for 8 months. They found that without antioxidant all $\mathrm{BH}_{4}$ was oxidized and that DTE was better at stabilizing $\mathrm{BH}_{4}$ than ascorbate (Fiege et al., 2004). Another study examined the stability of $\mathrm{BH}_{4}$ in blood at room temperature (Fekkes \& VoskuilenKooijman, 2007). It was found that $0.1 \%$ DTT offered better protection than 0.5 and $0.25 \%$ DTT. It was also observed that centrifugation of the blood sample right after it had been collected gave a lower total biopterin level compared to waiting for $3 \mathrm{~h}$ before centrifugation of the blood sample, and after $3 \mathrm{~h}$ the level of $\mathrm{BH}_{4}$ started to decline whereas the total biopterin level remained constant for 
24h (Fekkes \& Voskuilen-Kooijman, 2007). Our results do not show a higher biopterin level if the blood sample is allowed to stand for $3 \mathrm{~h}$ prior to centrifugation. However, if the methodology by Fekkes and Voskuilen-Kooijman is correctly described in their paper, it appears that the authors did not stoichiometrically add enough iodine to oxidize the added DTT. This could explain their findings since enough of the DTT could have reacted after 3 hours to leave sufficient iodine to oxidize $\mathrm{BH}_{4}$ and $\mathrm{BH}_{2}$ and thereby give a higher response.

Some of the observations that were made in the $\mathrm{BH}_{4}$ and $\mathrm{BH}_{2}$ model systems were also found in the blood samples whereas others were not. $\mathrm{BH}_{2}$ in model systems was degraded by acid whereas this did not seem to be the case in blood. The iodine oxidation method gives the total amount of $\mathrm{BH}_{2}$ and biopterin and if $\mathrm{BH}_{2}$ were converted exclusively to biopterin, this would not affect the measured amount. However, the model studies with $\mathrm{BH}_{2}$ clearly showed (Table 2) that $\mathrm{BH}_{2}$ is not only converted to biopterin but also to some unidentified species. Hence, if $\mathrm{BH}_{2}$ were degraded by acid in blood a lowering of the $\mathrm{BH}_{2}+$ biopterin level would have been expected. As this is not the case, protective factors such as endogenous ascorbate or EDTA coming from the blood sampling tubes are more likely to explain the findings. $\mathrm{BH}_{4}$ was found to degrade in model systems at pH 7.4 (Figure 3). This was not reproduced in blood samples. Even though degradation is slow $(0.25 \mathrm{nM} / \mathrm{h})$ about $1.5 \mathrm{nM}$, corresponding to $6 \%$, would have degraded in $6 \mathrm{~h}$ which would have been detectable. As above, the most likely reason is the presence of stabilizing factors in the blood samples such as ascorbate and EDTA. One significant finding that was observed in both model systems and blood samples was the lower level of $\mathrm{BH}_{4}$ with MPA, which seemed to be even worse in blood samples than in the model systems, and the lower recovery of total biopterins in blood samples (Table 3 ) and the $\mathrm{BH}_{2}$ model system (Table 2). TCA provided the highest level of $\mathrm{BH}_{4}$ and lowest level of $\mathrm{BH}_{2}$.

The reason for the differences in $\mathrm{BH}_{4}$ and $\mathrm{BH}_{2}$ levels observed with the three acids is not clear. The inverse relationship between $\mathrm{BH}_{4}$ and $\mathrm{BH}_{2}$ levels observed for the three acids (Table 3 ) could indicate different degrees of oxidation of $\mathrm{BH}_{4}$, i.e. most oxidation with MPA and least with TCA. However, levels of $\mathrm{BH}_{4}$ and $\mathrm{BH}_{2}$ did not change with time, which would have been expected if oxidation took place. Furthermore, the model studies showed that $\mathrm{BH}_{4}$ was not oxidized for $48 \mathrm{~h}$ in acid (with the exception of TCA with DTE). It therefore seems more likely that the acids interact differently with the iodine oxidation assay. MPA is unsuitable for biopterin analysis with the iodine oxidation assay, as the levels of $\mathrm{BH}_{4}$ are simply too low. Presumably, a higher $\mathrm{BH}_{4}$ level reflects less post-sampling oxidation, which leaves TCA the better choice as the level of $\mathrm{BH}_{4}$ is significantly higher and the level of total biopterins the same compared to PA (Table 3).
Comparing the different pretreatments of the samples, acid precipitation of the blood sample immediately after blood drawing seems to give the lowest level of $\mathrm{BH}_{2}$ and hence least oxidation (Table 4). However, the differences are small, albeit significant, and no method seems to be vastly superior to the others.

\section{Conclusions}

The high stability of $\mathrm{BH}_{4}$ and degradation of $\mathrm{BH}_{2}$ in acid and vice vers $a$ at neutral $\mathrm{pH}$ poses an analytical challenge when using the combined measurement as a biomarker of endothelial function. However, we have shown that the levels of $\mathrm{BH}_{2}+$ biopterin and $\mathrm{BH}_{4}$ did not change with time for up to $6.5 \mathrm{~h}$. TCA is the best choice for precipitating proteins whereas MPA should not be used. Whether plasma is separated and proteins precipitated immediately or at a later stage only has a minor effect on the levels of biopterins determined.

\section{Declaration of interest}

This work was supported by the LIFEPHARM in vivo pharmacology centre. The authors declared no conflict of interest.

\section{References}

Berka V, Yeh HC, Gao D, Kiran F, Tsai AL. (2004). Redox function of tetrahydrobiopterin and effect of L-arginine on oxygen binding in endothelial nitric oxide synthase. Biochemistry 43:13137-13148.

- Blair JA, Pearson AJ. (1973). A kinetic study of the autoxidation of tetrahydrobiopterin. Tetrahedron Lett 14:203-204.

Cañada-Cañada F, Espinosa-Mansilla A, Muñoz de la Peña A, Mancha de Llanos A. (2009). Determination of marker pteridins and biopterin reduced forms, tetrahydrobiopterin and dihydrobiopterin, in human urine, using a post-column photoinduced fluorescence liquid chromatographic derivatization method. Anal Chim Acta 648:113-122.

Crabtree MJ, Smith CL, Lam G, Goligorsky MS, Gross SS. (2008). Ratio of 5,6,7,8-tetrahydrobiopterin to 7,8-dihydrobiopterin in endothelial cells determines glucose-elicited changes in NO vs. superoxide production by eNOS. Am J Physiol Heart Circ Physiol 294:H1530-H1540.

Dántola ML, Vignoni M, Capparelli AL, Lorente C, Thomas AH. (2008). Stability of 7,8-dihydropterins in air-equilibrated aqueous solutions. Helv Chim Acta 91:411-425.

Davis MD, Kaufman S, Milstien S. (1988). The auto-oxidation of tetrahydrobiopterin. Eur J Biochem 173:345-351.

Espinosa-Mansilla A, Munoz de la Pena A, Canada-Canada F, Mancha de Llanos A. (2008). LC determination of biopterin reduced forms by UV-photogeneration of biopterin and fluorimetric detection. Talanta 77:844-851.

Feillet F, Clarke L, Meli C, Lipson M, Morris AA, Harmatz P, Mould DR, Green B, Dorenbaum A, Giovannini M, Foehr E; Sapropterin Research Group. (2008). Pharmacokinetics of sapropterin in patients with phenylketonuria. Clin Pharmacokinet 47:817-825.

Fekkes D, Voskuilen-Kooijman A. (2007). Quantitation of total biopterin and tetrahydrobiopterin in plasma. Clin Biochem 40:411-413.

Fiege B, Ballhausen D, Kierat L, Leimbacher W, Goriounov D, Schircks B, Thöny B, Blau N. (2004). Plasma tetrahydrobiopterin and its pharmacokinetic following oral administration. Mol Genet Metab 81:45-51. 
Fukushima T, Nixon JC. (1979). Oxidation and conversion of reduced forms of biopterin. In: Kisliuk RL and Brown GM (eds). Chemistry and Biology of Pteridines. New York, NY: Elsevier North Holland, Inc., 31-34.

Fukushima T, Nixon JC. (1980). Analysis of reduced forms of biopterin in biological tissues and fluids. Anal Biochem 102:176-188.

Heales SJ, Blair JA, Meinschad C, Ziegler I. (1988). Inhibition of monocyte luminol-dependent chemiluminescence by tetrahydrobiopterin, and the free radical oxidation of tetrahydrobiopterin, dihydrobiopterin and dihydroneopterin. Cell Biochem Funct 6:191-195.

- Heller R, Unbehaun A, Schellenberg B, Mayer B, Werner-Felmayer G, Werner ER. (2001). L-ascorbic acid potentiates endothelial nitric oxide synthesis via a chemical stabilization of tetrahydrobiopterin. J Biol Chem 276:40-47.

Howells DW, Hyland K. (1987). Direct analysis of tetrahydrobiopterin in cerebrospinal fluid by high-performance liquid chromatography with redox electrochemistry: prevention of autoxidation during storage and analysis. Clin Chim Acta 167:23-30.

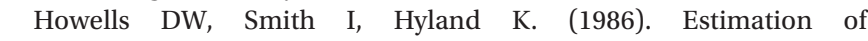
tetrahydrobiopterin and other pterins in cerebrospinal fluid using reversed-phase high-performance liquid chromatography with electrochemical and fluorescence detection. J Chromatogr 381:285-294.

Kar S, Kavdia M. (2011). Modeling of biopterin-dependent pathways of eNOS for nitric oxide and superoxide production. Free Radic Biol Med 51:1411-1427.

Katoh S, Akino M. (1966). In vitro conversion of sepiapterin to isosepiapterin via dihydrobiopterin. Experientia 22:793-794.

Lykkesfeldt J. (2000). Determination of ascorbic acid and dehydroascorbic acid in biological samples by high-performance liquid chromatography using subtraction methods: reliable reduction with tris[2-carboxyethyl]phosphine hydrochloride. Anal Biochem 282:89-93.

Lyudnikova T, Dashina O, Telegina T, Kritsky M. (2009). Investigation of the photochemical properties of biopterin and its reduced forms. Appl Biochem Microbiol 45:104-109.

Maharaj G, Selinsky BS, Appleman JR, Perlman M, London RE, Blakley RL. (1990). Dissociation constants for dihydrofolic acid and dihydrobiopterin and implications for mechanistic models for dihydrofolate reductase. Biochemistry 29:4554-4560.

- Mochizuki S, Ono J, Yada T, Ogasawara Y, Miyasaka T, Kimoto M, Kashihara N, Kajiya F. (2005). Systemic nitric oxide production rate during hemodialysis and its relationship with nitric oxide-related factors. Blood Purif 23:317-324.
Noguchi K, Hamadate N, Matsuzaki T, Sakanashi M, Nakasone J, Uchida T, Arakaki K, Kubota H, Ishiuchi S, Masuzaki H, Sugahara K, Ohya Y, Sakanashi M, Tsutsui M. (2011). Increasing dihydrobiopterin causes dysfunction of endothelial nitric oxide synthase in rats in vivo. Am J Physiol Heart Circ Physiol 301:H721-H729.

Pearson AJ, Blair JA. (1975). Autoxidation of tetrahydropterins. In: Pfleiderer W (eds). Chemistry and Biology of Pteridines. Berlin: Walter de Gruyter, 775-779.

Pearson AJ. (1974). Kinetics and mechanism of the autoxidation of tetrahydropterins. Chemistry and Industry, 233-239.

Pfleiderer W. (1982). Properties of naturally-owccurring pteridines. In: Wachter H, Curtius H-C, Pfleiderer W (eds). Biochemical and Clinical Aspects of Pteridines 1[Cancer, Immunology, Metabolic Diseases]. Berlin: Walter de Gruyter, 3-26.

Powers AG, Young JH, Clayton BE. (1988). Estimation of tetrahydrobiopterin and other pterins in plasma by isocratic liquid chromatography with electrochemical and fluorimetric detection. J Chromatogr 432:321-328.

- Presta A, Siddhanta U, Wu C, Sennequier N, Huang L, Abu-Soud HM, Erzurum S, Stuehr DJ. (1998). Comparative functioning of dihydroand tetrahydropterins in supporting electron transfer, catalysis, and subunit dimerization in inducible nitric oxide synthase. Biochemistry 37:298-310.

-Schircks B, Bieri JH, Viscontini M. (1978). Über Pterinchemie. 65. Mitteilung [1]. Herstellung von (6R,S)-5,6,7,8-TetrahydroL-biopterin, 7,8-Dihydro-L-biopterin, L-Sepiapterin, Deoxysepiapterin, (6R,S)-5,6-Dihydrodeoxysepiapterin und 2'-Deoxybiopterin. Helv Chim Acta 61:2731-2738.

- Takeda M, Yamashita T, Shinohara M, Sasaki N, Takaya T, Nakajima K, Inoue N, Masano T, Tawa H, Satomi-Kobayashi S, Toh R, Sugiyama D, Nishimura K, Yokoyama M, Hirata K, Kawashima S. (2009). Plasma tetrahydrobiopterin/dihydrobiopterin ratio: a possible marker of endothelial dysfunction. Circ J 73:955-962.

-Valent S, Tóth M. (2006). Activation energy determinations suggest that thiols reverse autooxidation of tetrahydrobiopterin by a different mechanism than ascorbate. Int J Biochem Cell Biol 38:1786-1793.

-Vásquez-Vivar J, Martásek P, Whitsett J, Joseph J, Kalyanaraman B. (2002). The ratio between tetrahydrobiopterin and oxidized tetrahydrobiopterin analogues controls superoxide release from endothelial nitric oxide synthase: an EPR spin trapping study. Biochem J 362:733-739.

Werner ER, Blau N, Thöny B. (2011). Tetrahydrobiopterin: biochemistry and pathophysiology. Biochem J 438:397-414. 